\title{
Fear Inducer: A Mixed Reality Audio Experience
}

\author{
Jurgen Westerhoff \\ Eindhoven university of Technology, Eindhoven
}

\begin{abstract}
In this paper I present the results from a design research project done at the Eindhoven University of Technology. In this project I explored the possibilities of mixing realities using sound. With this mixed reality I explored ways to make people scared for entertainment purposes, comparable to horror-movies or thrill rides. One of the main elements of the concept was to make the device mobile, so that a thrilling experience can be generated at any time and any place. The user will put on the audio-device and through a biofeedback-loop connect himself to the system. While walking around sounds come from the system that blend in with sounds from the real environment. This effect causes interesting situations, directed by the users own impulses sensed by the biofeedback-loop.
\end{abstract}

Keywords: mixed reality, audio experience, thrilling experience, biofeedbackloop.

\section{Introduction}

Recent trends in horror cinema show that there is a need for more extreme content and experiences in certain audiences. This is probably due to saturation of the audience,[1] and a need for new and exciting experiences. In this project different ways to scare people for entertainment purposes were explored, and how this can be done most effectively. Using a 'research through design' approach the user-experience was investigated with simple prototyping to see how people react to 'scary' stimuli.

Mixed reality [2] systems offer ways to blend the real with the virtual, and hereby open up a lot of possibilities for entertainment products. Most of the mixed reality systems however are focussed on visual effects[3][4], but audio has much potential as well. In this project the use of mixed reality in audio is investigated through the design of a product for a specific target group.

A large part of the effects induced by scary movies is related to instinctive reactions in the brain [5]. Apparently, the acted events in a movie trigger instinctive reactions and put the viewer in a state of 'fight or flight' just as real life situations would. This heightened state of arousal has some measurable effects on the human body, such as a change in heart-rate and sweating.

\section{Blending Real and Virtual}

\subsection{Letting the Imagination Run Wild}

One of the reasons that horror movies are getting boring for audiences is that the location and predictability of the scripts are often the same. A movie fan will be able to 
recognize style-elements and will get used to the familiar location of the theatre. All these things prohibit the imagination of the audience to run wild, this usually happens only after the movie. The opportunities lie in this after-effect of the movie, what happens when you keep on giving scary stimuli in this phase? The combination of subtle stimuli and the imagination of the audience could lead to tailored and non-scripted experiences.

This is where mixed reality starts to become interesting. In stead of the fixed setting of the theatre, there are now possibilities to combine virtual information with real world settings. The challenge is to feed the imagination with stimuli, and take away the boundary between what is real and what is virtual.

\subsection{Auditive Stimuli}

Auditive stimuli are very effective in inducing fear [5]. They affect a part of the brain that is quickly startled. This is an evolutionary warning system, used to alert you when unknown sounds appear. This is especially true when the context is unknown and the sounds are not easily recognized. Because of this primal reaction, audio is very suited to make people scared. This is also the reason why scary movies often have a very heavy sound-score.

Another plus-side of using auditive stimuli is that they blend seamlessly with realworld sounds (depending on the type of headphones). It keeps the field of view unhindered, and leaves room for imagination. This makes it ideal for a product in which freedom of movement is needed.

\subsection{Biofeedback}

Blending the virtual sounds with the real world poses some challenges. One of which is how to now when a person reacts to the sounds in the environment. With visual stimuli it is possible to track where a person is looking, and how he manipulates the real world. With sound however it is much harder to know which sounds have an effect on someone. To get an idea about which sounds have an effect on a person, I introduced a biofeedback system. This system uses galvanic skin response to sense the stress-levels of the user. By doing this the system is able to react on objects in the surroundings of the user, and adjusting to that. So if for instance the user is walking past a dark corridor and the stress levels rise, the system is able to activate a scary sound to react to the reaction of the user. This creates a loop in which the experience of the user activates virtual sounds which in their turn influence the perception of the real world.

This use of biofeedback creates the link between the virtual sounds and the stimuli coming from the real world. The resulting effect is that in the perception of the user the sounds coming from the system and the sounds from the real world blend together.

\subsection{The Scare Effect}

This blending of real and virtual is what makes the experience scary. During the user tests it became apparent that this state (of blending) is not immediately in effect. At first the difference between the virtual and the real world is distinguishable, but after a while the devision starts to become vague. Especially abstract sounds are hard to 
pinpoint. Things like the wind or a barking dog are hard to place in either the virtual or real world. The resulting effect is a state in which the user does not know where sounds are coming from, and this gives a very menacing and scary experience. You can imagine for instance that when a virtual sound of a barking dog starts coming towards you, you cannot help but look behind you to check if this is not happening in the real world. This effect intensifies over time while using the system. Test-subjects expressed that the final minutes of the test were scariest because they had become paranoid of all the sounds that came from the system.

\section{The Fear Inducer System}

To test the effects of the audio-experience a fully working prototype was made. This prototype consisted out of three elements; a headset, a biofeedback loop and a series of audio-samples. The user puts the headset on, and walks around outside. Fig.1 shows a short scenario of how the system could be used. The system is able to detect rises in stress-levels, and will play a scary audio sample as a reaction to that. The prototype was tested on a small group of test-subjects, who had to walk a given route in a context unknown to them. This test was highly experimental, and only gave an impression on how the subjects experienced the prototype. The results from this test showed that the first impressions of the subjects was very positive, they experienced it as very thrilling and were willing to try it again.

I will shortly describe the workings of the separate elements, and how they affect the overall experience of using the prototype.
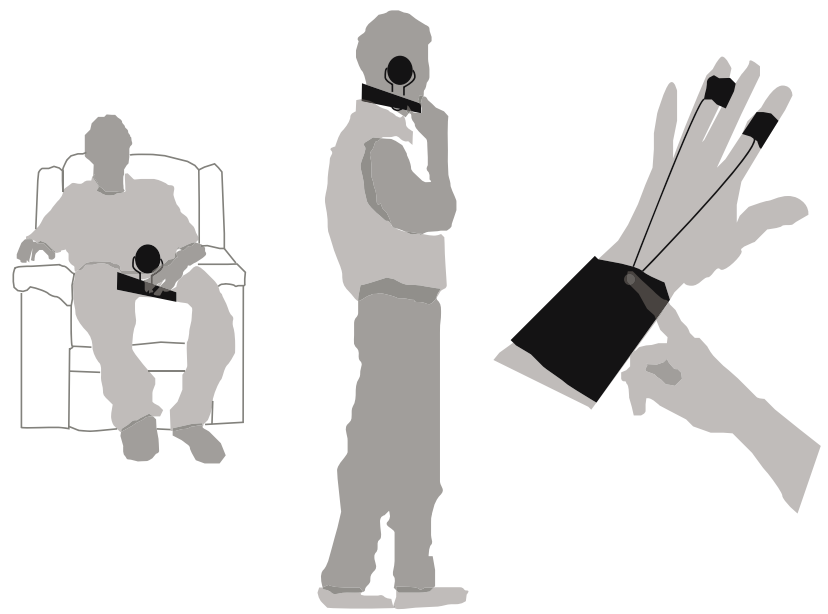

Fig. 1. Putting on the headset and biofeedback system

\subsection{Design of the Headset}

Next to the auditive stimuli, the headset itself also contributes to the experience. Throughout a series of pilot tests it became clear that not being able to look behind you 
has a big effect on how scary people perceived a context. This passive haptic feedback does nothing more than restrict head-movements. The effect however on the overall experience is considerable. Next to this restriction the overall design of the headset is quite menacing, and looks unpleasant to put on (actually wearing the headset however is quite comfortable). Fig.2 shows a picture of the final prototype headset. These design-choices came directly from several levels of user involvement throughout the project. The overall look and feel were inspired by a persona [7] that was made about the targeted user group. The challenge was to make it fit with other products used by the target group, without having to lean to much on classical horror-themes.

\subsection{The Biofeedback Loop}

The biofeedback system works by measuring the galvanic skin response(gsr) of the user. When the user is in a stressful state, the system will be able to read this and give back a value. It basically is a very simple electronic circuit in which the user is used as a variable resistor. In the prototype the gsr-measurement is done at the fingertips, because this gives to clearest reading.

To explore how much the body reacts to scary stimuli, a small test was performed. Several test-subjects were placed in front of a screen and attached to a biofeedback measurement circuit (measuring gsr). On the screen a scene from a recent horror-movie was shown, and the gsr-readings were recorded on a computer. At scary parts in the movie the gsr-reading clearly showed peaks, about three seconds after the actual scary scene. This delay is probably a sum of the lag in sweat building up, and the processing of the sensor-reading. Because the readings gave clear enough results to detect peaks in scariness, the system proved itself robust enough for use in a prototype.

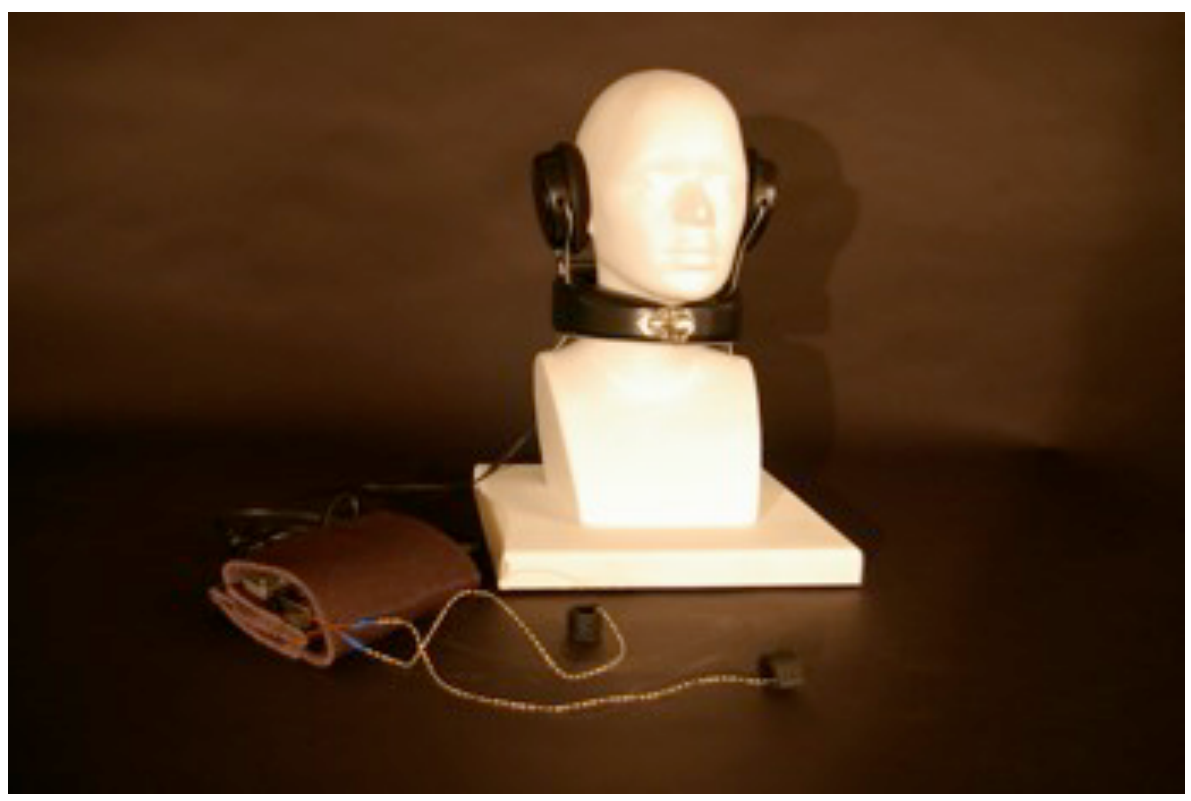

Fig. 2. The final prototype headset 


\subsection{The Audio}

In the prototype the audio was stored and played on two separate mp3-players. These players were connected to the biofeedback system, and would play a song when a certain threshold in the biofeedback-readings was reached. The audio samplers were divided in two types, one mp3-player held the ambient sounds and the other one the audio-effects. By using two players, the audio was mixed seamlessly.

The ambient sounds would play continuously, and increased in volume and intensity during the length of the test. This created a suspense, not unlike what is used in movies[8]. The sound effects however were only played when a peak in the biofeedback readings was measured (what meant that the test-subject was excited by something). The sound effects ranged from barking dog sounds to more abstract howling sounds. During the user-test the subjects expressed that the more abstract sounds that seemed to be coming towards them (with virtual $3 \mathrm{~d}$ sound techniques) were scariest.

\section{Conclusion}

Through this paper I attempted to describe the progress I made with showing the possibilities of mixed reality using audio. Although the project was highly experimental, I hope to show the potential of mixed reality in audio through the form of a product. One of the advantages of the 'research through design' approach is that you can take the wishes and reactions of real people into the process. The reactions of people to the final prototype, and the involvement of users in early explorations in the project helped the development take big steps in a short amount of time. With this paper I want to show an interesting direction of design research, and hope to encourage people to develop more entertainment devices using the possibilities of mixed reality.

\section{References}

1. Rauterberg, M.: About a framework for information and information processing of learning systems. In: Falkenberg, E., Hesse, W., Olive, A. (eds.) Information System Concepts Towards a consolidation of views, pp. 54-69 (1995)

2. Milgram, P., Kishino, F.: A taxonomy of mixed reality visual displays. IECE Transactions on Information Systems E77-D(12) (December 1994)

3. Cho, K., Kang, W., Soh, J., Lee, J., Yang, H.S., Hunter, G.: A handheld augmented reality game system with dynamic environment. In: Ma, L., Rauterberg, M., Nakatsu, R. (eds.) ICEC 2007. LNCS, vol. 4740, pp. 10-15. Springer, Heidelberg (2007)

4. Abawi, D.F., Reinhold, S., Dorner, R.: A Toolkit for authoring non-linear storytelling environments using mixed reality. In: Göbel, S., Spierling, U., Hoffmann, A., Iurgel, I., Schneider, O., Dechau, J., Feix, A. (eds.) TIDSE 2004. LNCS, vol. 3105, pp. 113-118. Springer, Heidelberg (2004)

5. Thompson, B.: Evoking Terror In Film Scores. M/C: A Journal of Media and Culture 5.1 (2002), http://www.media-culture.org.au/0203/evoking.php 
6. LeDoux, J.: The Emotional Brain: The Mysterious Underpinnings of Emotional Life. Touchstone, New York (1996)

7. Olsen, G.: Persona Creation and Toolkit (2004),

http: / / www. interactiondesigners.com

8. Sirish, K.A.: Computational model of suspense in virtual worlds, Raleigh (2003) 